\title{
СКАНИРУЮЩАЯ ЗОНДОВАЯ МИКРОСКОПИЯ 2D НАНОРАЗМЕРНЫХ СТРУКТУР ДЛЯ ЭНЕРГОНАКОПИТЕЛЕЙ И КАТАЛИЗАТОРОВ
} SCANNING PROBE MICROSCOPY OF 2D NANOSTRUCTURES FOR ENERGY STORAGE AND CATALYSTS

\begin{abstract}
И.В.Яминский', 2,3, д.ф.-м.н., проф., генеральный директор Центра перспективных технологий, директор Энергоэффективных технологий (ORCID: 0000-0001-8731-3947), А.И.Ахметова 1, 2, 3, инженер НИИ ФХБ имени А.Н.Белозерского МГУ, ведущий специалист Центра перспективных технологий и Энергоэффективных технологий (ORCID: 0000-0001-6363-8202), Г.Б.Мешков', к.ф.-м.н., ст. науч. сотрудник (ORCID: 0000-00033930-3730), А.В.Оленин', к.Х.н., ст. н. сотрудник (ORCID: 0000-0003-1376-2090) / yaminsky@nanoscopy.ru I.V.Yaminsky', 2,3, Doctor of Sc. (Physics and Mathematics), Prof., Director of Advanced Technologies Center, Director of Energy Efficient Technologies, A.I.Akhmetoval, 2,3, Engineer of A.N. Belozersky Institute of Physico-Chemical Biology, Leading Specialist of Advanced Technologies Center and of Energy Efficient Technologies, G.B.Meshkov', Cand. of Sc. (Physics and Mathematics), Senior Researcher, A.V.Olenin', Cand. of Sc. (Chemistry), Senior Researcher
\end{abstract}

DOI: 10.22184/1993-8578.2019.12.2.148.151

Получено: 22.03.2019 г.

В рамках работ по модификации строения и определения физико-химических и электрофизических характеристик 2D наноразмерных структур проведено исследование их проводимости. Получены данные об электрической проводимости структур $\mathrm{TiS}_{3}$ на поверхности оксида кремния. Измерены проводимости графена и графита методом сканирующей резистивной микроскопии. Данные получены с помощью сканирующего зондового микроскопа ФемтоСкан.

As a part of the work to modify the structure and determine the physicochemical and electrophysical characteristics of 2D nanoscale structures, their conductivity was studied. The data on the electrical conductivity of $\mathrm{TiS}_{3}$ structures on the surface of silicon oxide were obtained. The conductivity of graphene and graphite was measured by resistive scanning microscopy. The data were obtained using a FemtoScan scanning probe microscope.

$\mathbf{M}$ ногие из хорошо изученных 2D-материалов относятся к семейству халькогенидов переходных металлов [1, 2, 3]. Большинство этих материалов в объемном виде имеет слоистую структуру со слабыми межслоевыми Ван ${ }^{-}$ дер-Ваальсовыми взаимодействиями. До настоящего времени экспериментальные исследования были в основном сосредоточены на таких материалах, как $\mathrm{MoS}_{2}, \mathrm{MoSe}_{2}, \mathrm{WS}_{2}$ и $\mathrm{WSe}_{2}$. Однако, семейство 2D-материалов очень богато и содержит много других слоистых материалов с интересными свойствами, которые в настоящее время получили ограниченное внимание исследователей. Одним из таких перспективных материалов является трисульфид титана $\left(\mathrm{TiS}_{3}\right)$. Трисульфид титана (сернистый титан) является слоистым полупроводниковым соединением, которое образует кристаллы моноклинной сингонии.

Сканирующая зондовая микроскопия (С $3 \mathrm{M})$ позволяет получать данные не только о топографии поверхности образца, но и оценить величину электрической проводимости областей 


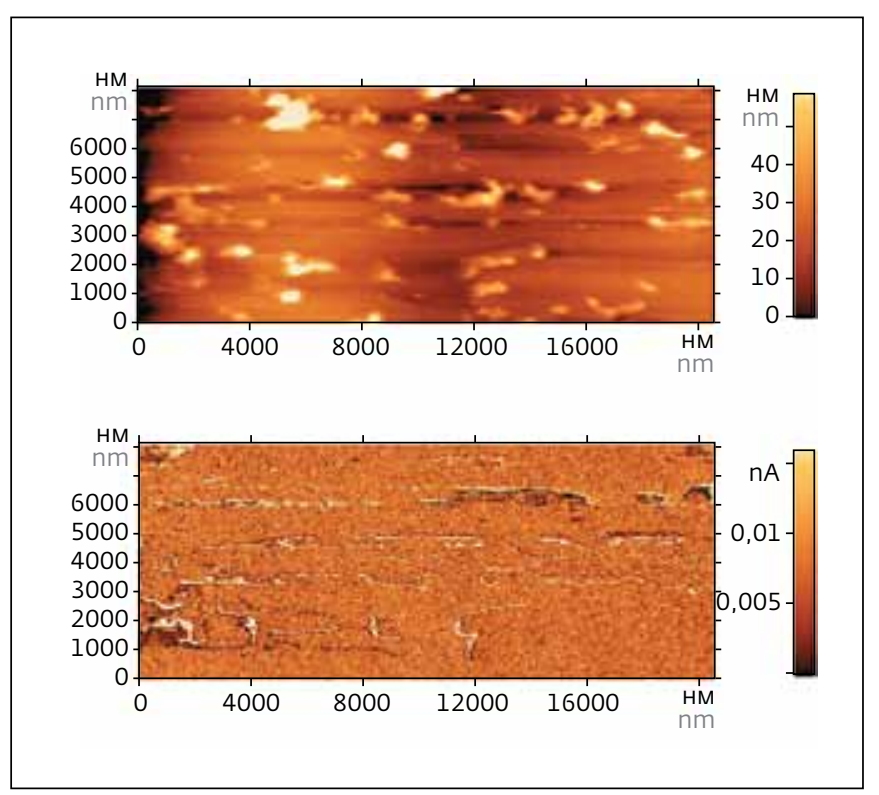

Рис.1. Изображение поверхности пленки трисульфида титана в режиме сканирующей резистивной микроскопии. Верхнее - изображение топографии поверхности, нижнее - карта распределения тока, протекающего через кантилевер в данной области

Fig.1. The image of titanium trisulfide film surface in the scanning resistive microscopy. The upper image shows the surface topography, the lower image presents the map of current distribution flowing through the cantilever in this area

в нанометровом масштабе [4]. Также с помощью СЗМ можно установить взаимосвязь между локальной структурой (геометрией и физикохимическим составом) поверхности углеродных и 2D-материалов с их электрофизическими свойствами [5]. Повышение быстродействия зондовой микроскопии позволяет существенно улучшить временное разрешение и наблюдать в режиме реального времени многие процессы на поверхности материалов, используемых в накопителях и преобразователях энергии. Применение новых режимов сканирования, в которых осуществляется прецизионное контролируемое перемещение зонда по трем координатам в пространстве с использованием систем обратной связи, позволяет исследовать материалы с очень сильно развитой поверхностью [6].

Для определения электрической проводимости поверхности образца сернистого титана использовался режим сканирующей резистивной микроскопии с применением кантилеверов с проводящим покрытием (марка HA-CNC-AU, ScanSens $\mathrm{GmbH}$, Германия). Величина приложенного напряжения между образцом и проводящим кантилевером составляла 0,5 В. Вариации тока находились в диапазоне до 150 пА, что позволяет оценить проводимость образца менее $10^{5} \mathrm{Om} / \mathrm{cm}$.

При измерении проводимости графена на поверхности $\mathrm{SiO}_{2}$ (толщиной 300 нм) наблюдаются неоднородности поверхности. На поверхности образца существуют проводящие области (графен) и не проводящие (оксид кремния). Данные, полученные в режиме топографии, показывают, что поверхности графена и кремния имеют схожий рельеф. В областях, где образец не проводит, иногда наблюдаются выступающие одиночные или множественные "рыхлые" пики.

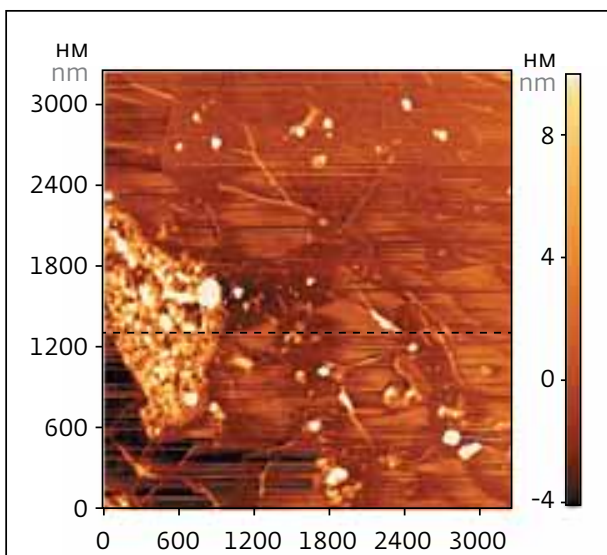

a)

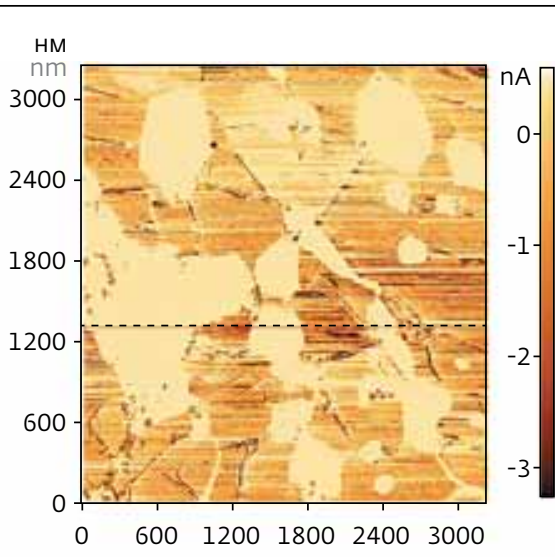

b)
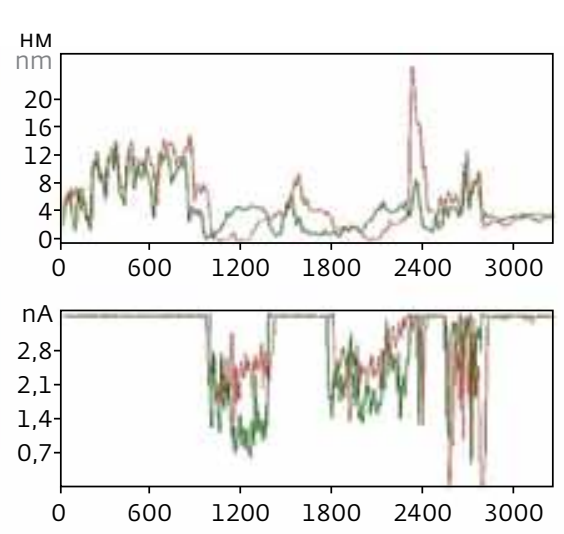

C)

Pис.2. a-moпография графена, b-проводимость. График (с) показывает зависимости высоты (верхняя часть) и проводимости (нижняя часть) в выбранном сечении, соответствующем пунктирной линии на изображениях $а$ и $b$

Fig.2. $a$ - topography of graphene, $b$ - conductivity. The graph shows the dependences of the height (upper part) and conductivity (lower part) in the selected section corresponding to the dashed line in the $a$ and $b$ images 


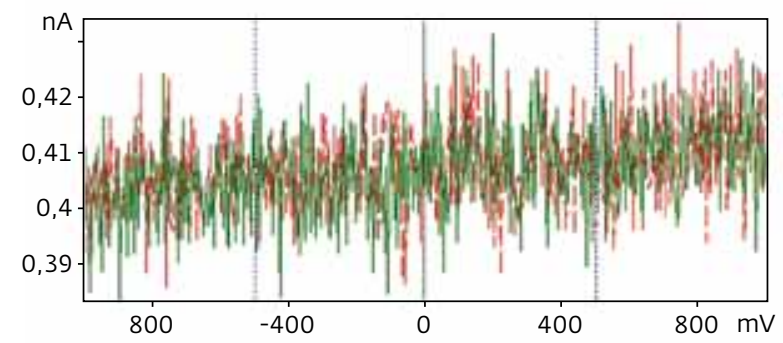

a)

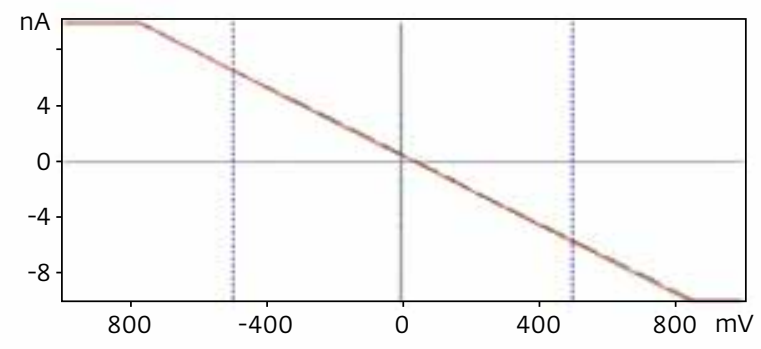

b)

Рис.3. ВАХ на поверхности кремния (a) и графена (b)

Fig.3. VA characteristics on the silicon surface (a) and graphene (b)
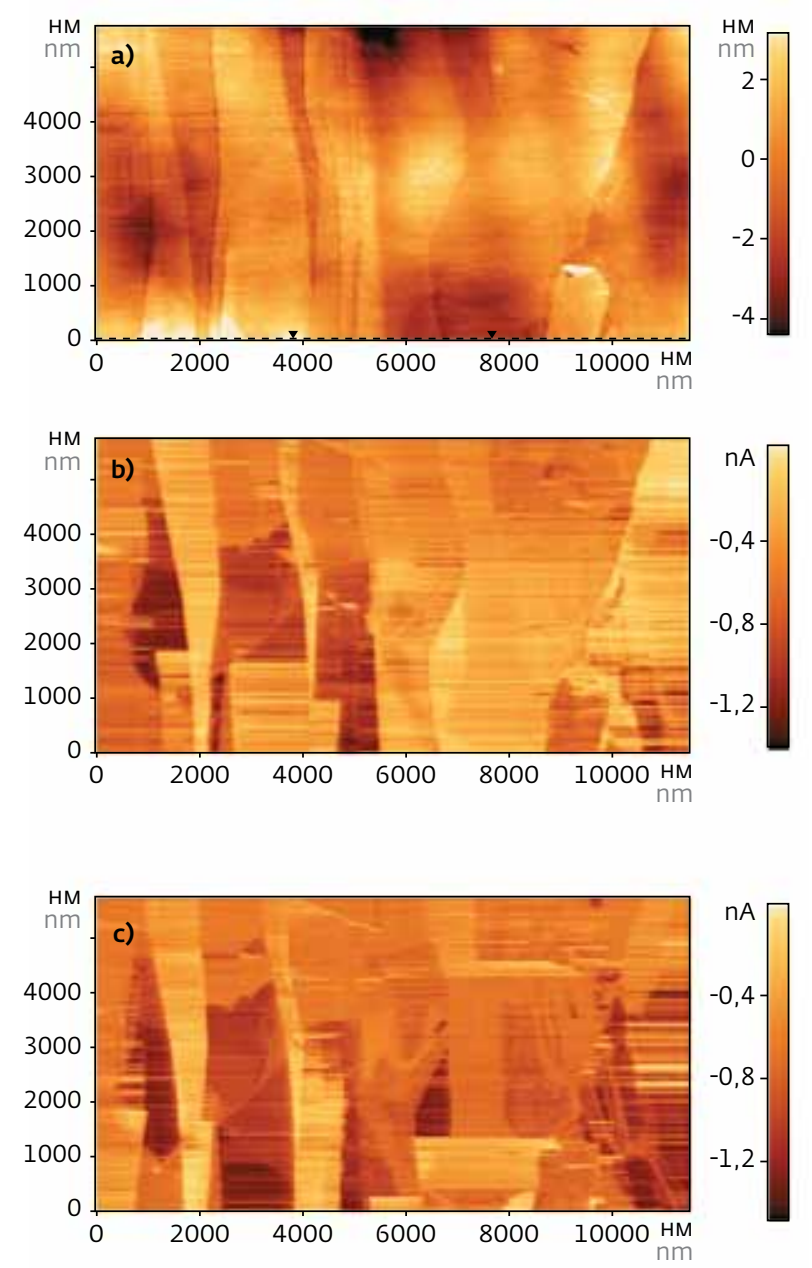

Pис.4. $a$ - monография графита, $b$ - проводимость графита при движении зонда слева направо, с - справа налево Fig.4. $a$ - topography of graphite, $b$-graphite conductivity when probe moving from left to right and $c$ - from right to left
Предполагается, что поверхность образца не является абсолютно "чистой", на ней находятся частицы, нарушающие проводимость. Указанное соответствие легко наблюдать в горизонтальном сечении (рис.2).

Проводящие свойства графена и кремния также подтверждаются следующим экспериментом. В произвольных точках поверхности измерялись вольтамперные характеристики (BAX). Если точка попадала на непроводящий участок, то BAX представляла собой шум вблизи нуля. Если же точка попадала на графен, наблюдалась строгая линейная зависимость, проходящая через ноль (рис.3).

При измерении проводимости графита наблюдается корреляция топографии поверхности и ее проводящих свойств. Слои проводят практически однородно, но на их границах наблюдаются резкие скачки проводимости. Как правило, вышележащие слои проводят лучше. Контакт зонда с образцом не является стабильным и изменяется при переходе с одной террасы на другую. Об этом свидетельствует сильное различие данных, полученных при сканировании справа налево и слева направо (рис.4). Изменение наблюдаемой картины проводимости образца при смене направления сканирования, а также характер выбросов, наблюдаемых между террасами, связаны, по всей видимости, с асимметрией и изменением проводящего покрытия на зонде.

Также проводились измерения проводимости графита на никеле. Данный образец изготавливался при высокой температуре, после чего остывал. Вследствие этого при остывании образец сжимался, и на поверхности образовывались характерные складки (рис.5).

Как мы видим из представленных данных, сканирующая зондовая микроскопия является информативным инструментом при измерении проводимости поверхности и позволяет оценивать физические свойства образца при количественном 


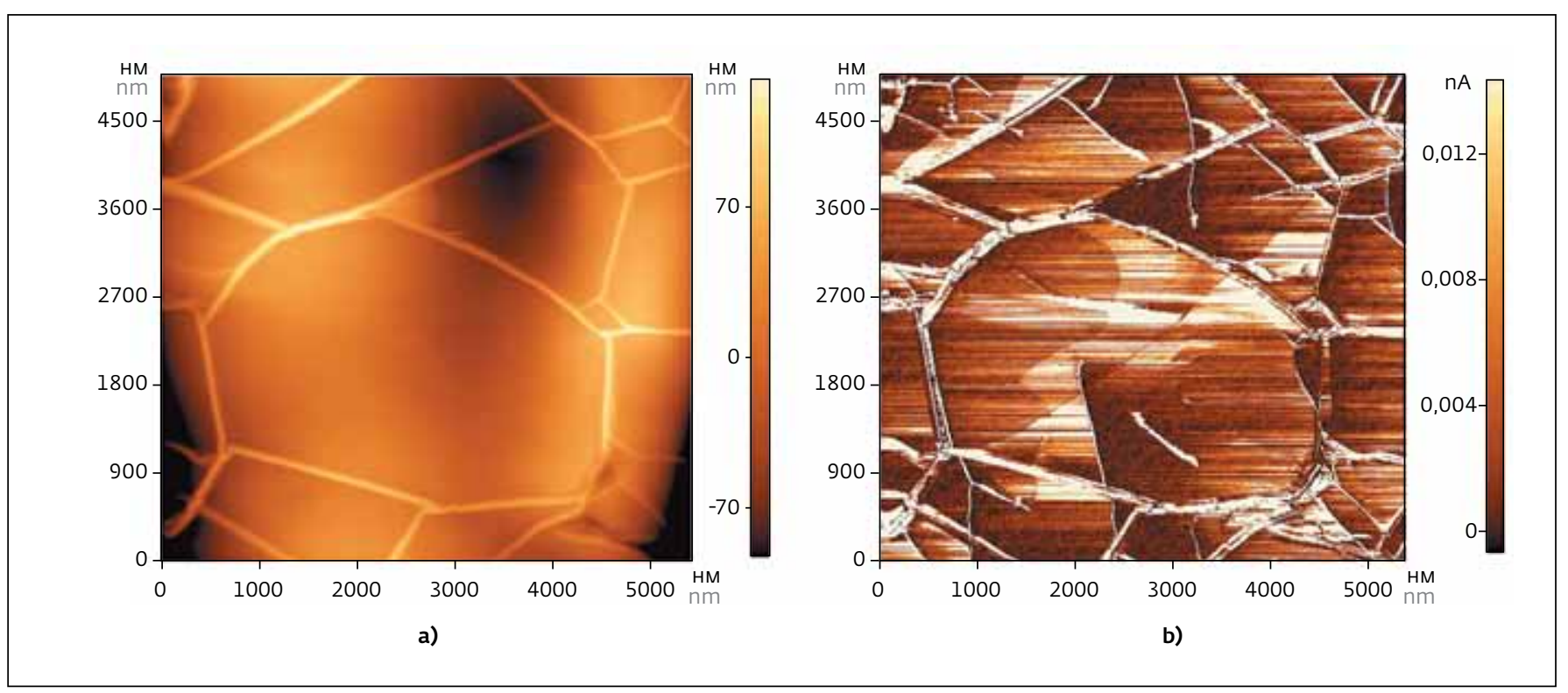

Puc.5. Топография (a) и проводимость (b) графена на никеле Fig.5. Topography (a) and conductivity (b) of graphene on nickel

сравнении данных топографии поверхности и данных проводимости.

Исследование выполнено при финансовой поддержке РФФИ в рамках научного проекта 16-29-06290.

\section{ЛИTEPATУPA / REFERENCE}

1. Wang Q.H., Kalantar-Zadeh K., Kis A., Coleman J.N., Strano M.S. Nat. Nano. 2012. 7. $699-712$.

2. Chhowalla M., Shin H.S., Eda G., Li L.-J., Loh K.P., Zhang H. Nat. Chem. 2013. 5. 263-275.

3. Jariwala D., Sangwan V.K., Lauhon L.J., Marks T.J., Hersam M.C. ACS Nano. 2014. 8. 1102-1120.
4. Sinitsyna O., Akhmetova A., Meshkov G., Goncharova T., Pylev I., Smirnova M., Belov Y., Yaminsky I. The effect of the graphite precursor microstructure on the formation of graphite oxide // Nanoindustry, 2 (81): 170-172, 2018.

5. Akhmetova A.I., Meshkov G.B., Sinitsyna O.V., Yaminsky I.V. Nanoscopy methods for directional modification of nanoscale 2D structures and determining physicochemical and electrophysical characteristics // Nanoindustry. 2018. T. 3. No. 83. P. 246-249.

6. Yaminsky I.V., Akhmetova A.I., Belov Yu.K. Nanotokar // Nanoindustry. 2018. Vol. 10. No. 6 (85). P. 446-448.

\section{8-Я МЕХДУНАРОДНАЯ КОНФЕРЕНЦИЯ ПО НАНОТЕХНОЛОГИЯМ, KИTAЙ (CHINANANO 2019)}

В Пекине (Китай) 17-19 августа 2019 года состоится 8-я Международная конференция по нанотехнологиям (ChinaNANO 2019). Завоевав всемирную известность, ChinaNANO стала универсальной платформой для научных дискуссий, промышленного сотрудничества и стратегического планирования. В этом году она соберет новаторов и участников из всех частей мирового сообщества в области науки и нанотехнологий, чтобы они поделились научными и технологическими достижениями в разных секторах промышленности, новыми перспективами и возможностями. Состоятся форумы для обсуждения влияния и ответственности науки перед обществом. Заслуженные ученые и пионеры отрасли будут председательствовать на тематических сессиях, чтобы стимулировать диалог по возникшим в этих областях проблемам. Будут созданы специальные подфорумы, в том числе и 3-й Форум по нанотехнологиям и наукоемким отраслям, Национальный форум по науке и симпозиумы, организованные совместно с выдающимися академическими организациями для развития академических и деловых связей, взаимодействия науки и промышленности, будущего сотрудничества и партнерских отношений, а также поощрения молодых ученых, которые поделятся результатами своих исследований, в содействии их карьерному росту.

По мamepuanam: http://www.chinanano.org 